\title{
PROBLEM SOLVING AND DECISION MAKING
}

\author{
Chaired by Darrell Butler, Ball State University
}

\section{A real-time data collection system for problem-solving protocols}

\author{
SHARON A. R. KRISTOVICH \\ University of Illinois, Chicago, Illinois
}

\begin{abstract}
A computer program was developed to collect solution protocols with written explanations, with a level of detail difficult to obtain with traditional pencil-and-paper tasks. The program automatically presents the instructions, problems, solution entered, changes to the solution, explanation prompts, solution feedback, and system help in a Windows environment. The time to make each move and type each explanation is recorded, making it possible to compare solution times across treatment groups. Protocols are stored in individual ASCII files, eliminating transcription, and a summary data set for all protocols is created. The program was tested in a study where individual protocols were collected in small groups. This program can readily be adapted to different experimental contexts and can be a useful educational tool.
\end{abstract}

In cognitive psychology, particularly problem-solving research, verbal self-report protocols are used with increasing frequency to detect strategies, level of understanding, and transfer of training. Typically, subjects are asked to verbalize everything they are thinking while solving a problem. This "think-aloud" report supplements any written solution information, provides a representation of a subject's problem-solving session, and is thought not to affect the cognitive processes involved in solving the problem (Ericsson \& Simon, 1980, 1994). More recently, the uses of verbal reports in problem-solving protocols have been extended to include verbal explanations for actions taken while problem solving (e.g., AhlumHeath \& DiVesta, 1986; Berardi-Coletta, Buyer, Dominowski, \& Rellinger, 1995; Gagné \& Smith, 1962). This reason-giving procedure differs from the think-aloud procedure in that thinking aloud often does not produce justifications for actions taken during problem solving. Verbal explanations, however, do appear to affect cognitive processing, since giving verbal explanations during practice problem solving has been found to improve performance on subsequent isomorphic tasks with no requirements to state reasons (Ahlum-Heath \& DiVesta, 1986; Berardi-Coletta et al., 1995; Gagné \& Smith, 1962).

This paper is based on a part of S.A.R.K.'s doctoral dissertation. The author greatly appreciates the comments of David Kristovich on earlier versions of this paper. Correspondence should be addressed to S. A. R. Kristovich, 1709 Gentry Square Lane 101, Champaign, IL 61821 (e-mail: kristo@sundog.sws.uiuc.edu).
Verbal explanations given after the practice problems were correctly solved also improved performance on problems presented later (Kristovich, 1988), and the types of verbal explanations produced for worked-out examples have been found to distinguish high-performing from low-performing students (Chi \& Bassok, 1989; Chi, Bassok, Lewis, Reimann, \& Glaser, 1989). Thus, generating verbal explanations for actions can alter thinking quality, with interesting empirical and educational implications.

While solution protocols may provide valuable data for the scientist and educator, the time-consuming task of collecting them often makes data collection impractical. Data must be collected individually, often resulting in fewer subjects used, which may ultimately affect generalizability. Furthermore, the researcher's constant presence may improve performance (Davis, Carey, Foxman, \& Tarr, 1968). Also, solution protocols with verbal explanations can produce additional data collection problems. Simultaneously taping protocols, managing multiple stopwatches, recording data, and monitoring subjects can be cumbersome. While the verbal portion of the protocol can be tape-recorded and transcribed, this method delays analysis and adds time, expense, and the potential for mistakes. Often, measurements such as the time it takes to make each move and generate each explanation, which could be powerful learning indicators, must be consolidated into a total solution time. For example, a reduction in the time to make each move (not including the time spent giving explanations) may demonstrate im- 
proved problem understanding and move compilation. For these reasons, a program was developed that provides an improved method of subject protocol data collection. This program was designed to present a variety of problem types and to collect protocols with or without explanations. This system presents either one problem, as in baseline studies, or multiple problems in sequence, as in studies on transfer of training.

\section{The Program}

A computer program, written in Turbo Pascal 6.0, was developed to address the concerns surrounding individual protocol collection and to allow for small-group data collection, with each subject using his/her own computer. The program was designed to run in MS-DOS mode on an IBM-PC compatible computer, with at least a 286 processor and a minimum of $640 \mathrm{~K}$ RAM. The program automatically presents the instructions, problems, solutions entered, reason prompts, solution feedback, and system help in a Windows environment.

First, a window appears with some initial instructions about the program and how the answers are to be entered. After reading the instructions at their own pace, users are first given two practice questions to enter answers and then given the first problem to solve. Throughout the solution, the problem descriptions remain on the screen, and smaller windows for the solution, responses, and help appear when appropriate. In all instances, the windows remain on screen as long as necessary, and the user closes the window by pressing the Esc key. These smaller windows are sometimes subdivided into smaller "boxes" when multiple responses are required. For example, in some of the problem solution windows, each solution component is entered in a separate box. Users can enter the solution components in any order they wish by pressing the letter assigned to the box they wanted to use, typing their entry, then pressing the Enter key to record it. Every instance of pressing Enter is recorded as a "move" (all instances of pressing the Enter keys without an entry are discounted later). Users can also "scratch out" or restart their problem solution any time by pressing Alt-R. Restarting the solution erases any material in the answer box, and the protocol file is marked with the text "Restart," with all previous solution attempts remaining in the file.

Feedback is given only when all the problem requirements are met, or the solution attempt is clearly wrong, whichever comes first. For correct solutions in all problem classes, a window announces a correct answer and the next task appears when the user presses a key. For incorrect solutions, the feedback window was customized to the type of problem being solved. The feedback window disappears when any key is pressed, and the user can then make corrections or restart his/her solution until it is correct. On-screen help can be obtained at any point in the program by pressing the F1 key, and the help windows are tailored for the state of the program in use. For instance, if a subject is uncertain about how to answer one of the practice questions, the help window will give $\mathrm{him} /$ her detailed instructions on how to enter his/her answer. Any access to the help feature is included in the total time to solve the problem, and a recent revision of the program records the total time spent using the help feature, by task. Finally, each subject's protocol is stored in a separate ASCII file, eliminating the need for transcription, and the written explanations are matched to the entry for which they were given. In addition, the program produces a summary data file that lists the total moves, solution time, and time to type explanations for every protocol collected to date. This file can be loaded directly into a statistical software package.

With this program, more data can be obtained than with traditional data collection methods. First, using the program allows for data collection with groups of subjects working individually. Second, the program records the entire problem state, not just the specific move made, eliminating the need for transcription. Third, the time to generate each entry, as well as the time spent giving reasons, is recorded. This way, the time spent giving explanations can be subtracted from the total time spent solving the problem, allowing for solution time comparisons across treatment groups.

\section{An Example of the Program in Use}

The original objective of the program was to collect solution protocols for a study that examined the effect that giving explanations has on problem-solving performance and the transfer of these effects to novel tasks. This study's design provided ideal circumstances to test the system. Three experiments were conducted, employing the same mixed design, in which three practice problems were administered, followed by three transfer problems in one of three treatment conditions. Each experiment had three different sets of practice problems, which were based on Greeno's (1978) three problem classes: Transformation, Inducing Structure, and Arrangement. Each problem set contained four isomorphic and successively more difficult problems, and the first three versions in each set were used as practice problems. The three transfer problems consisted of the hardest versions from each problem class, and were identical for all three experiments. For example, in the first experiment, the Transformation problems served as the three practice problems and one transfer problem, while the remaining two transfer problems were the most difficult problems from the Arrangement and Inducing Structure classes. The presentation order of the last two problems were counterbalanced by the program. Baseline data were collected for each problem in each class so that practice effects in the main experiments could be estimated. For this, the program administered and recorded one problem only, without prompting for explanations.

The Transformation problems were a variation of Luchins's (1942) water jars problems, called the "sandcontainer" problem. The goal of the sand-container prob- 


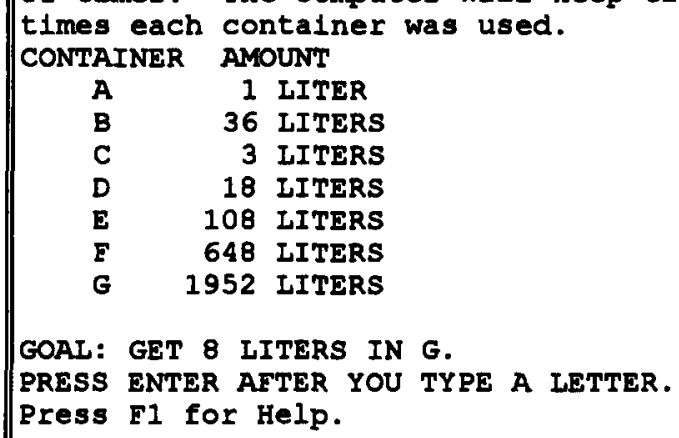

Figure 1. An example of the transfer Transformation problem as it appeared to subjects on the computer.

lem was to reduce sand in a container to $8 \mathrm{~L}$ by using each of a set of containers (1) at least once and (2) the fewest number of times. Figure 1 shows how the transfer sandcontainer problem appeared to subjects. The subject entered the letter identifying the container that he/she wished to use. Since this problem was designed to be difficult to solve "in the head," the program computed how much sand remained in the initial container after each move and the number of times each container was used. For this problem, feedback consisted of whether the attempt was "correct but inefficient" (and therefore not the correct solution), or "incorrect" (when multiple solution requirements were not met). Program solutions were automatically restarted when an attempt was clearly incorrect; that is, if less than the goal amount was left in the container; if the goal amount was obtained but the containers were not all used, or if the solution strategy was not the most efficient one. Subjects could not change a previous move but could restart their attempt.

The Inducing Structure problem class was represented by matrix problems similar to the ones used by Schwartz (1971) and Polich and Schwartz (1974). In these problems, a series of statements or clues was presented, and subjects were asked to determine the relationships among

In the problem below, three men are in a hospital. Each one is suffering from a different disease, and is in a different room. Using the statements below as clues, figure out which men have which disease and what room they are in. Complete the chart in the box in the lower right corner. The words or parts of words in capital letters (e.g., OSBORN) are the items that can be typed in the answer box. All the room numbers can be typed in the answer box. Press the LETTER to the RIGHT of the box to get to the bOX. PRESS ENTER AFTER PLACING EACH WORD IN A BOX. Press F1 for Help.

1. THOMAS, OSBORN and WILSON have either ASTHMA, MEASLES or EPILEPSY and are either in rooms 101,102 or 103 .

2. WILSON is in room 102 .

3. The man with EPILEPSY is in room 103.

4. THOMAS has ASTHMA.

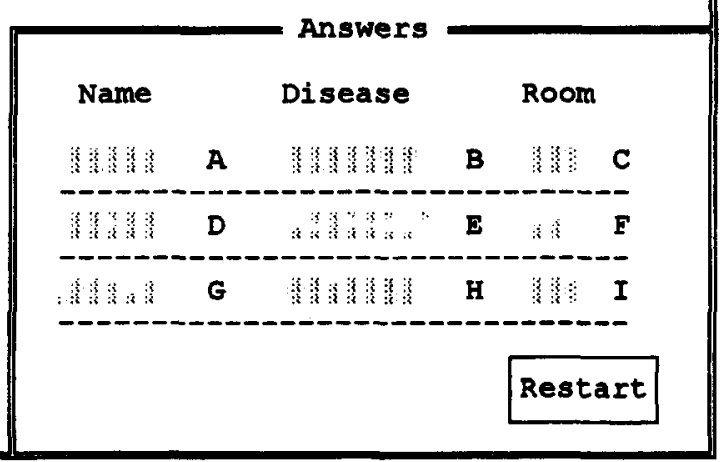

Figure 2. An example of the first practice Inducing Structure problem as it appeared to subjects on the computer. 
them. Figure 2 shows how the first practice problem initially appeared to subjects. This problem had three classes with three items in each class: hospital room number $(101,102$, and 103), patient's name (Thomas, Osborn, and Wilson), and disease (asthma, measles, and epilepsy). Subjects were given the four clues shown in Figure 2 and asked to detect the relationships among the items across classes. Subjects had continuous access to the clues and their partial solution. The columns in the on-screen matrix were already labeled with the problem's object classes, and spaces beneath each column were for each item. Rows contain related items across classes, such as "Thomas, asthma, and 101" in Figure 2. Subjects entered only one item at a time and received solution feedback (correct or not) only after all the boxes in the grid were completed. When solutions were incorrect, changes were made to the grid until the correct solution was found.

A variation of Katona's (1940) card problem, used by Berardi-Coletta (1990; Berardi-Coletta et al., 1995), represented Arrangement problems. The goal of the card problem is to arrange a set of cards to appear in a predetermined order when dealt in a specified fashion. To deal the cards in this problem, the first card was dealt face up and the next card was placed on the bottom of the deck, continuing until all cards were face up. For the practice problems, subjects were instructed to arrange numbered cards so they would appear in ascending order (e.g., 2, 3, $4,5,6,7)$ when dealt. For the transfer problem, subjects were instructed to arrange eight cards, four red and four black, so they appeared alternating red and black when dealt. Figure 3 shows how the transfer card problem appeared to subjects on the screen. Once all the valid cards were "arranged" or placed in the boxes, the program "dealt" the cards, showing the subject the order (from left to right) in which the cards appeared "face up." Feedback was given every time the complete deck was arranged, for illegal responses (such as entering a number twice), and incorrect solution attempts. Changes to the solution were made in the same manner as the other problem classes, one box at a time, until the correct solution was obtained.

Three experimental treatments were used in this study. One group, reasons-during, was asked to give explanations, or reasons for moves, immediately after making them. Subjects in another group, reasons-after, were required to give explanations for actions once they finished solving each practice problem. The third group was a practice-only control condition where subjects did not give any explanations. For the reasons-during treatment, once a move was entered, a window prompting, "Why did you make this move?" appeared. It was removed from the screen only after subjects typed an explanation. The same window appeared for reasons-after subjects, after the problem was correctly solved, while the program replayed each move. No explanation window appeared for the practice-only group. Solution protocols were obtained for 270 experimental subjects, each solving six problems ( 30 for each of three treatment groups in each of three experiments). In addition, 360 baseline protocols ( 30 subjects for each of the 12 problems used) were collected. Thus, the design size and quantity of data made it exceedingly difficult for individual protocol collection. Furthermore, this design could provide a test of the presentation of multiple problems of various types, with different task demands.

\section{Sample Protocols}

Each problem set had different on-screen representations, and the forms of the solution protocols in the data set were different. The following is an example of a partial Transformation practice problem protocol for a reason-during subject:




$0221 \mathrm{D} \mathrm{f}$

1110110010000

0221163 because I want to pour 3 liters of B into C which makes 11 liters in $B$

1278101010000

0221257 because I need to eliminate more liters to get a total of 8 liters in B

Note there are only 10 liters left in B.

The first line of the protocol (022 $1 \mathrm{D} \mathrm{f})$ is identifying information, listing subject id (022), experiment number (1), treatment condition (D), and gender (f). The next line contains a partial answer, or problem state placed in the protocol file when the subject pressed the Enter key (1 110110010000 ). The first digit is the problem number (1); the second digit is the move number, or when in the sequence a partial solution or move was created (1); and the third set of digits is the time it took to make the partial solution in seconds (10). Following the time to make the move is the problem state $\left(\begin{array}{llllll}11 & 0 & 0 & 0 & 0 & 0\end{array}\right)$. The move made is determined by comparing two consecutive problem states to each other to find the one item that has changed between them. In the protocol given here, the first number in the problem state is the sand remaining in the target container after the move was made. The remaining sets of digits are the number of times each container was used to obtain the 8 - $\mathrm{L}$ goal. The containers are labeled $A$ through $G$ to the subjects, reading from left to right. Therefore, in this sample protocol, the container A (with a 1-L capacity) was used, and the sand in the initial container was reduced from 11 to $10 \mathrm{~L}$.

A set of identifying information was listed before each explanation. For the explanation " 0221163 because I want to pour 3 liters of $B$ into $C$ which makes 11 liters in $B$," the first set of digits (022) is the subject number; the second (1) is the problem number; the third (1) is the move number for the explanation, and the last set of digits (63) is the time, in seconds, taken to type the explanation. The move number for the reason is the same as the move number in the protocol. Since reasons-during subjects gave explanations immediately after they made each partial solution, a line of text containing the explanation appears immediately after the partial solution.

The following is an example of the last two moves for one problem of a practice-only Inducing Structure protocol:

$\begin{array}{ll}18553 & \\ \text { Thomas Asthma } & 101 \\ \text { Wilson Measles } & 102 \\ \text { Osborn } & 103 \\ 19602 & \\ \text { Thomas Asthma } & 101 \\ \text { Wilson Measles } & 102 \\ \text { Osborn Epilepsy } & 103\end{array}$

This sample protocol is one entry (entering "Epilepsy" in the blank space) from a completed and correct solution, shown in the ninth move. These problems had multiple-line partial solutions because subjects placed their solutions in a matrix or grid on the screen. The first line identifies the problem number, the move number, and the solution time, as described earlier. After the first line, the partial solution is the matrix representation entered by the subject up to that point (seven entries were made previously).

It was found that $66 \%$ of the subjects (approximately one third in each treatment condition) made spelling errors when writing their responses into the answer boxes for the matrix problem. Thus, although the relationships were correct, spelling mistakes would result in solution errors by the program. For this study subjects were further instructed to be careful of spelling mistakes, and were monitored by the experimenter for spelling errors. Plans for this program include incorporating a spelling checker to check typed words in a solution.

The following is an example of a partial Arrangement problem protocol for a reasons-after subject:

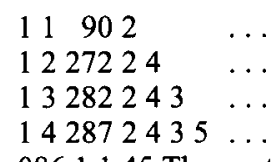

0861145 The next card needs to be a 3

0861242 The second card was placed on bottom and the next card needed to be a 3

0861354 Needed for the 5 card to come up last

0861457 Put 5 in the last box so it would come up last

Again, the first three sets of numbers in each line represent the problem number, move number, and time (in seconds) to make each move. Only one card changes in each partial solution. For instance, in the second line of the example, the subject placed the 4 in the second card slot. The rest of the solution is unchanged. The "." means that those slots were not available to use with that particular version of the problem. Reasons-after subjects gave explanations after the problem was solved and their protocol lists all explanations after the solution was listed. Again, for the explanations, the first set of digits (086) is the subject number; the second (1) is the problem number; the third is the move number; and the last set is the time (in seconds) taken to type the explanation.

Once the data were collected, the summary data were analyzed, and the explanations given were classified by type of process involved in making the statement. The protocol coding was done by hand, and it was soon realized that future development of this program should incorporate a tool to help coding and evaluating protocols. This tool would find a protocol, display a move and its explanation on the screen, and provide a box to record notations.

Very few computer-related problems occurred in the study, and those problems were not directly related to the program. Only 27 protocols needed to be recollected because of diskette failures that occurred after the data were collected. In addition, only 2 subjects abandoned the study because of their fear of computers, with the remaining subjects reporting they felt comfortable using the computer program. When appropriate, subjects complied with the instructions to give explanations, as $42 \%$ 
$55 \%$ of their explanations focused on the task, and $41 \%$ $50 \%$ focused on their cognitive processes used to achieve the solution. Thus, the system provided an efficient method of obtaining solution protocols where the experimenter's constant presence was not necessary.

\section{Issues Surrounding Computer Use}

While this system saved many hours of time and expense, several conceptual issues remain regarding the use of a computer to record problem-solving protocols. One issue is the imposition of a problem representation with the form of the answer box. First, it might provide a hint to a subject who otherwise has no idea how to approach the problem. Second, the imposed representation may make it more difficult to solve the problem for someone using a different internal representation. This is particularly true for the Inducing Structure problems, where several physical representations for the problem are possible (Schwartz, 1971). When the answer boxes were constructed and the data were collected, several steps were taken to keep these problems at a minimum. First, no clues or hints were given to subjects, and they were allowed to enter their answers in any order (any box), and to change their answers anytime. Second, a matrix format was used for the Inducing Structure problems. Schwartz (1971) found this to be the most frequently used format to solve this type of problem. No data exist on the modes of representation for these Transformation and Arrangement problems, so the most open-ended layouts were used in constructing the answer box.

Another issue is whether written explanations are adequate substitutes for verbal ones. Giving verbal reasons can be interpreted to mean that verbalization per se somehow improves understanding. This implication is not consistent with the existing research, however. First, much evidence shows that thinking aloud does not affect performance. Kristovich (1988) found that thinkingaloud subjects made the same number of mistakes on the practice and transfer Tower of Hanoi problems as a noverbalization control. However, giving verbal reasons in practice significantly reduced mistakes. Similar results were found by Berardi-Coletta (1990; Berardi-Colletta et al., 1995), Allwood (1990), and Johnson (1993). These findings lend support to the idea that the verbal component of verbal reason giving is not the critical component affecting performance. Second, research examining verbal versus nonverbal justifications has also shown that the verbal component is not critical. Wilder and Harvey (1971) found that giving reasons reduced the mistakes made on the six-disk Tower of Hanoi transfer task, but differences in mistakes were not found among the verbal versus nonverbal components. Dörner (1978) and BerardiColletta et al. (1995) found that nonvocal self-reflection improved the performance on later presented tasks. Dörner explained his findings by proposing a model where processing results can be redirected by the central processor to evaluative processes that determine its effectiveness. Similarly, Stanley, Mathews, Buss, and Kotler-Cope
(1989) and McGeorge and Burton (1989) argued that verbal knowledge comes after understanding the task, suggesting that understanding improves articulation rather than vice versa. Therefore, it seems unlikely that the overt, verbal component of reason giving is the crucial element in improving performance. Rather, the deliberate act of providing explanations seems responsible for the performance changes. However, compliance with the instruction to give reasons is difficult to verify without any report. As a compromise, written explanations were required in the present study to provide verification of the experimental manipulation without the overt, verbal component. In a future study, whether any performance differences exist between written versus oral reasons will be examined directly.

This program is currently designed to collect protocol data for a particular study; however, object-oriented programming makes it possible to incorporate new problems and experimental designs with a minimal amount of software development. Thus, this program can be easily adapted to meet a variety of experimental contexts. For instance, it would not be difficult to modify the program to include a delay of feedback variable, or to provide feedback after a fixed number of moves. New problems could be added by simply adding a new object to the code.

This software would also be a valuable educational tool. Since giving reasons seems to help improve performance both on practiced problems and novel ones, practice giving explanations with this program may improve classroom performance, with little instructor intervention. Instructors could therefore provide individualized, self-paced instruction, giving constructive feedback on a print copy of the protocol. Feedback could then focus on both solution methods (e.g., strategies) and more effective and appropriate explanations.

\section{REFERENCES}

Ahlum-Heath, M. E., \& DiVesta, F. J. (1986). The effect of conscious controlled verbalization of a cognitive strategy on transfer in problem solving. Memory \& Cognition, 14, 281-285.

ALLWOOD, C. M. (1990). On the relation between justification of solution method and correctness of solution in statistical problem solving. Scandinavian Journal of Psychology, 31, 181-190.

BERARDI-COLETTA, B. (1990). The effects of verbalization on problem solving and problem solution transfer-A metacognitive perspective. Unpublished doctoral dissertation, University of Illinois at Chicago.

Berardi-Coletta, B., Buyer, L. S., Dominowski, R. L., \& RellINGER, E. S. (1995). Metacognition and problem solving: A processoriented approach. Journal of Experimental Psychology: Learning, Memory, \& Cognition, 21, 205-223.

CHI, M. T. H., \& BAssoK, M. (1989). Learning from examples via selfexplanations. In L. B. Resnick (Ed.), Knowing, learning, and instruction: Essays in honor of Robert Glaser (pp. 251-282). Hillsdale, NJ: Erlbaum.

ChI, M. T. H., BassoK, M., Lewis, M. W., Reimann, P., \& Glaser, R. (1989). How students study and use examples in learning to solve problems. Cognitive Science, 13, 145-182.

Davis, J. M., Carey, M. H., Foxman, D. N., \& TARr, D. B. (1968). Verbalization, experimenter presence, and problem solving. Journal of Personality \& Social Psychology, 8, 299-302.

DöRNER, D. (1978). Self-reflection and problem solving. In F. Klix 
(Ed.), Human and artifical intelligence (pp. 101-107). Berlin: Deutscher Verlag der Wissenschaften.

Ericsson, K. A., \& Simon, H. A. (1980). Verbal reports as data. Psychological Review, 87, 215-251.

Ericsson, K. A., \& Simon, H. A. (1994). Protocol analysis: Verbal reports as data. Cambridge, MA: MIT Press.

GAGNÉ, R. M., \& SMITH, E. C., JR. (1962). A study of the effects of verbalizations on problem solving. Journal of Experimental Psychol$o g y, 65,12-18$.

GREENO, J. G. (1978). Natures of problem solving abilities. In W. K. Estes (Ed.), Handbook of learning and cognitive processes: Vol. 5 . Human information processing (pp. 240-269). Hillsdale, NJ: Erlbaum.

Johnson, M. M. S. (1993). Thinking about strategies during, before and after making a decision. Psychology \& Aging, 8, 231-241.

KATONA, G. (1940). Organizing and memorizing. New York: Columbia University Press.

KRISTOVICH, S. A. R. (1988). The effect of verbal report time and reason-giving on solution transfer. Paper presented at the annual meeting of the Midwestern Psychological Association, Chicago.
Luchins, A. S. (1942). Mechanization in problem solving the effect of Einstellung. Psychological Monographs, 54 (3, Whole No. 248).

MCGeorge, P., \& Burton, A. M. (1989). The effects of concurrent verbalization on performance in a dynamic systems task. British Journal of Psychology, 80, 455-465.

Polich, J. M., \& SchWarTZ, S. H. (1974). The effect of problem size on representation in deductive problem solving. Memory \& Cognition, 2, 683-686.

SCHWARTZ, S. H. (1971). Modes of representation and problem solving: Well evolved is half solved. Journal of Experimental Psychology, 91, 347-350.

Stanley, W. B., Mathews, R. C., Buss, R. R., \& Kotler-Cope, S. (1989). Insight without awareness: On the interaction of verbalisation, instruction and practice in a simulated process control task. Quarterly Journal of Experimental Psychology, 41A, 553-577.

WILDER, L., \& HARVEY, D. J. (1971). Overt and covert verbalization in problem solving. Speech Monographs, 38, 171-176.

(Manuscript received November 13, 1995; revision accepted for publication January 22, 1996.) 